\title{
Joint CoMP-Cell Selection and Resource Allocation in Fronthaul-Constrained C-RAN
}

\author{
Lei You ${ }^{1}$ and Di Yuan ${ }^{1,2}$ \\ ${ }^{1}$ Department of Information Technology, Uppsala University, Sweden \\ ${ }^{2}$ Department of Science and Technology, Linköping University, Sweden \\ \{lei.you; di.yuan\}eit.uu.se
}

\begin{abstract}
Cloud-based Radio Access Network (C-RAN) is a promising architecture for future cellular networks, in which Baseband Units (BBUs) are placed at a centralized location, with capacity-constrained fronthaul connected to multiple distributed Remote Radio Heads (RRHs) that are far away from the BBUs. The centralization of signal processing enables the flexibility for coordinated multi-point transmission (CoMP) to meet high traffic demand of users. We investigate how to jointly optimize CoMP-cell selection and base station resource allocation so as to enhance the quality of service $(\mathrm{QoS})$, subject to the fronthaul capacity constraint in orthogonal frequency-division multiple access (OFDMA) based C-RAN. The problem is proved to be $\mathcal{N} \mathcal{P}$. hard in this paper. To deal with the computational complexity, we derive a partial optimality condition as the foundation for designing a cell-selection algorithm. Besides, we provide a solution method of the optimum of the time-frequency resource allocation problem without loss of fairness on the QoS enhancement of all users. The simulations show good performance of the proposed algorithms for jointly optimizing the cell selection and resource allocation in a C-RAN, with respect to QoS.
\end{abstract}

\section{INTRODUCTION}

\section{A. Background}

Mobile networks are evolving rapidly in terms of coverage, capacity and new features, continuously pushed by new requirements related to latency, traffic volumes and data rates [1]. The operators are seeking for promising ways to increase the flexibility of cellular infrastructures so as to simplify the deployment and management of the network integrated with different communication technologies, e.g. mmWave, Li-Fi, coordinated multipoint transmission (CoMP), etc. [1]. Cloudbased Radio Access Network (C-RAN), referring to the virtualization of base station functionalities by means of cloud computing, results in a novel cellular architecture that costefficiently enables centralization and cloudization of largescale cooperative signaling processing in a network-wide manner [2], and thus reducing the overall network complexity [3] in respect of management and deployment.

In a C-RAN, the functions of Baseband Units (BBUs) and Remote Radio Heads (RRHs) are redefined, with some of the BBU processing functions being shifted to the RRH, which leads to a change in the BBU and RRH architecture [4]. The BBUs are aggregated in a pool and co-located in a Radio Cloud Center (RCC), and the RRHs that are separately distributed away from the RCC form Radio Remote Systems (RRSs). The link connecting a BBU with an RRH is referred to as fronthaul. One advantage of C-RAN lies in its ability to implement the CoMP transmission among multiple RRHs, based on its centralization of signal processing [4]-[6]. On the other hand, CoMP may result in more data traffic on fronthaul, which means that the CoMP performance depends on the capacity of fronthaul [4]-[6]. With the limited fronthaul capacity and bit-rate demands of user equipments (UEs), the CoMP performance benefits from optimizing the association pattern between RRHs and UEs.

\section{B. Motivation}

Several literature has focused on the CoMP techniques in CRAN. In [7], the authors studied the CoMP-based interference mitigation in heterogeneous C-RANs deployed with small cells. In [8], the authors investigated the Joint Transmission (JT) CoMP performance in C-RANs with large CoMP cluster sizes. Also, it is shown by [8] that CoMP transmission can be efficiently and effectively implemented based on the cooperation of a limited set of stations forming a so-called "CoMP cluster" in a C-RAN. The authors in [9] investigated the resource allocation of CoMP transmission in C-RAN, and proposed a fairness-aware user-centric scheme for enhancing the network coverage and achievable rate. In [10], state-of-theart and challenges of heterogeneous C-RANs are surveyed. The authors showed that limited fronthaul capacity affects the CoMP performance, pointing out that optimal resource allocation solutions call for investigation under the fronthaul capacity constraint. In [11], the authors studied jointly cellselection and resource allocation problems, in C-RANs of non-CoMP case. In [12], the resource allocation problem was studied in OFDMA-based C-RANs, with the framework of small cell underlaying a macro cell.

We remark that our work is motivated by the research mentioned above, as all of them stressed the benefits on network performance improvement by optimization of cell selection as well as resource allocation in C-RANs. On the other hand, so far we have not found literature that studies how to jointly optimize the cell selection and network-wide time-frequency resource allocation of CoMP transmission in C-RANs. From our perspective, in the CoMP scenarios, it is more crucial to optimize the time-frequency resource allocation subject to the constraints on QoS demands compared to the non-CoMP case, as CoMP introduces more dependencies of resource consumption and control signaling among cells/stations involved in cooperations. Also, under the capacity limit of fronthaul, the QoS satisfaction is highly affected by the CoMPcell selection along with the resource allocation strategies. 


\section{Contributions}

In this paper, we study how to jointly optimize the CoMPcell selection and the time-frequency resource allocation among cells, subject to the limit of fronthaul capacity, in order to maximize the fairness-aware QoS. We prove the $\mathcal{N} \mathcal{P}$ hardness of the problem, and provide theoretical insights as foundation of designing an efficient cell selection algorithm. As for the resource allocation, we derive a solution method with respect to optimizing QoS performance, subject to the QoS fairness constraints among all users. We proved that the solution method for resource allocation achieves the optimum, under any chosen CoMP-cell selection. Also, we show theoretically that the proposed CoMP-cell selection algorithm and the resource allocation method are naturally combined with each other, which is shown numerically to achieve good performance on fairness-aware QoS.

\section{SySTEM MODEL}

\section{A. General Description}

The setting of the system model is coherent with [4]. We consider a C-RAN with a centralized RCC, which has $n$ BBUs in the BBU pool. Each BBU is connected with several RRHs by fronthaul. This BBU and its connected RRHs form a socalled "C-RAN Cluster", and is referred to as "cluster" in this paper for simplicity. The system model is illustrated in Figure 1. We consider JT-CoMP. Within a cluster, the BBU calculates the coordinated beamformer for each RRH and all co-clustered RRHs can be jointly coordinated to serve UEs. Without loss of generality, we assume that each RRH is located on one Base Station (BS). Downlink transmission is studied in this paper.

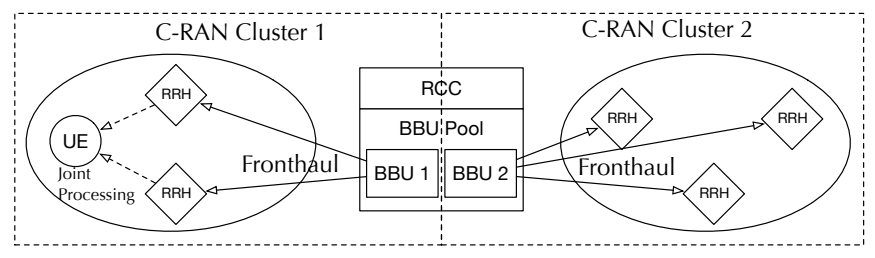

Figure 1. System model illustration.

\section{B. Basic Notations}

Denote by $\mathcal{C}=\{1,2, \ldots, n\}$ the set of C-RAN clusters. Denote by $\mathcal{R}=\{1,2, \ldots, m\}$ the set of RRHs in the C-RAN. Denote by $\mathcal{J}=\{1,2, \ldots, q\}$ the set of UEs. Since each cluster has only one BBU and there is no shared BBU among clusters, we also use $1,2, \ldots, n$ to refer to the corresponding RRHs for the $n$ clusters, respectively. For the same reason, we use $1,2, \ldots, m$ to refer to the corresponding BSs located with the $\mathrm{n}$ RRHs as well as the corresponding fronthaul, respectively. To avoid being ambiguous, the network entities that we refer to by using the indexes, will be explicitly clarified, in accordance with the context. Denote by $c_{i}$ the capacity of the fronthaul connected to RRH $i(i \in \mathcal{R})$. Denote by $\ell(i)$ and $\ell(j)$ the cluster in which RRH $i(i \in \mathcal{R})$ and $U E j(j \in \mathcal{J})$ located, respectively. For each UE $j$, denote by $\mathcal{J}_{j}$ the set of BSs/RRHs serving
UE $j$ via CoMP. We use the symbol $\circ$ to denote function composition.

\section{CoMP Transmission}

Denote by $p_{i}$ the transmit power of BS $i, i \in \mathcal{R}$. Denote by $h_{i j}$ the channel gain between BS $i$ and UE $j$. Denote by $\boldsymbol{w}_{i}$ the precoder of BS $i$. Let $\boldsymbol{x}$ be the channel input symbol sent by the cooperating BSs $\mathcal{J}_{j}$. Entity $\boldsymbol{x}_{k}$ denotes the channel input symbol sent by the other cells that are not cooperatively serving UE $j$. The received channel output at UE $j$ can be written as

$$
\mathbf{s}=\sum_{i \in \mathcal{J}_{j}} \sqrt{p_{i}} \mathbf{h}_{i j}^{\mathrm{H}} \boldsymbol{w}_{i} \boldsymbol{x}+\sum_{k \in \mathcal{R} \backslash \mathcal{J}_{j}} \sqrt{p_{k}} \mathbf{h}_{k j}^{\mathrm{H}} \boldsymbol{w}_{k} \boldsymbol{x}_{k}+\sigma
$$

Assuming that $x$ and $x_{k} k \in \mathcal{R} \backslash \mathcal{J}_{j}$ are independent zeromean random variables of unit variance, the SINR of UE $j$ is given by the equation as below [13],

$$
\gamma_{j}=\frac{\left|\sum_{i \in \mathcal{J}_{j}} \sqrt{p}_{i} h_{i j}^{H} w_{i}\right|^{2}}{\sum_{k \in \mathcal{R} \backslash \mathcal{J}_{j}} p_{k} I_{k j}+\sigma^{2}}
$$

where we have $I_{k j}=\left|h_{k j}^{H} \boldsymbol{w}_{k}\right|^{2} \rho_{k}$ and $\rho_{k}$ is the indicator of that whether $j$ receives interference from BS $k$.

For the sake of presentation, we use the binary matrix $\kappa \in\{0,1\}^{\mathrm{m} \times \mathrm{q}}$ as the indicator for the association relationship between BSs and UEs, by defining $\mathcal{J}_{j}(j \in \mathcal{J})$ as a mapping of $\kappa$ to a set of $\mathrm{BS}(\mathrm{s})$, as shown in (3). The obligation of each cluster for providing service to UEs is clarified in (4).

$$
\begin{gathered}
\mathcal{J}_{j}:\{0,1\}^{\mathrm{m} \times \mathrm{q}} \rightarrow 2^{\{1,2, \ldots, \mathrm{m}\}}: \kappa \mapsto\left\{i: \mathrm{K}_{i j}=1\right\} \\
\kappa_{i j}=0 \quad \ell(i) \neq \ell(j)
\end{gathered}
$$

\section{Resource Allocation}

Consider in OFDMA any fixed short period $T=\left[\tau_{s}, \tau_{e}\right]$ of a few hundred milliseconds, with $\tau_{s}$ and $\tau_{e}$ be the starting and ending time points, respectively. Without loss of generality, we use the term "Resource Unit" (RU) as the minimum unit for OFDMA time-frequency resource allocation. Suppose that in each BS the total number of RUs is $M$. For the cell(s) serving UE $j(j \in \mathcal{J})$, we denote $\alpha_{j}$ as the proportion of allocated RUs for transmission to UE $j$ over the total number $M$ of RUs in each BS. (Note that in JT-CoMP, the BSs that are cooperatively serving one UE use the same time-frequency resource for transmission to the $\mathrm{UE}$, and thus there is no need to use index $\alpha_{i j}$ and $\alpha_{k j}$ for differentiating BS $i$ and $k$, $i, k \in \mathcal{J}_{j}$.) For the network-wide resource allocation, we have $\alpha=\left[\alpha_{1}, \alpha_{2}, \ldots, \alpha_{\mathrm{q}}\right]^{\top}$. There is no specific bias on resource allocation for transmission, and thus each RU has the same opportunity used by BS for serving UE. The term $\sum_{j \in \mathcal{J}_{k}} \alpha_{j}$ computes the proportion of occupied RUs in BS k. For any RU that is being used for transmission, the probability that it is interfered by another BS $\mathrm{k}$ (assuming $\mathrm{k}$ is not a cooperative BS in this transmission) is $\sum_{j \in \mathcal{J}_{k}} \alpha_{j}$. For characterizing the influence of resource allocation on the inter-cell interference, we let $\rho_{k}$ be the mapping $\rho_{k}: \mathbb{R}_{+}^{q} \rightarrow \mathbb{R}_{+}: \alpha \mapsto \sum_{j \in \mathcal{J}_{k}} \alpha_{j}$ in (2). Also, $\rho_{k}$ is named as the load of BS $k$, for any $k \in \mathcal{R}$. 
Denote by $B$ the bandwidth per RU. The achievable bit rate of a (JT) link to UE $j$ can be calculated by $C_{j}: \mathbb{R}_{+}^{q} \rightarrow \mathbb{R}: \alpha \mapsto$ $M B \log \left(1+\gamma_{j} \circ \rho(\alpha)\right)$ according to the Shannon's capacity. We assume the data traffic requested by $\mathrm{UE} j(j \in \mathcal{J})$ takes place with density $t_{j}$ over time period $T$, and $t=\left[t_{1}, t_{2}, \ldots, t_{q}\right]$. As for ensuring the allocated RUs to $U E j$ is sufficient for transmission, we should have in (5) that

$$
\alpha_{j} \geqslant \int_{T} \frac{\eta_{j} t_{j}(\tau)}{C_{j}(\alpha, \kappa)} d \tau
$$

where $\eta_{j}$ is UE $j$ 's bit rate scaling parameter over the time period $T$. In other words, $\int_{T} \eta_{j} t_{j}(\tau) d \tau$ is the data traffic volume transmitted to UE $j$ scaled by $\eta_{j}$ over period $T$. Network-widely, we have $\eta=\left[\eta_{1}, \eta_{2}, \ldots, \eta_{n}\right]^{\top}$. As for the constraint of limited resource and fronthaul capacity, (6) holds, where $\bar{\rho}$ is the maximum resource limit of BSs.

$$
\sum_{j \in \mathcal{J}_{i}} \int_{T} \eta_{j} t_{j}(\tau) d \tau \leqslant c_{i} \text { and } \rho_{i}(\alpha, \kappa) \leqslant \bar{\rho} \quad i \in \mathcal{R}
$$

Note that $\eta_{j}$ reflects the "QoS satisfaction" of UE $j$. In other words, $\eta_{j}$ indicates how well the traffic demand of UE $j$ is satisfied, under the constraints (5) and (6). Besides, we take into consideration the fairness of QoS satisfaction among UEs. The metric Jain's Fairness is used for evaluation, i.e. $J: \mathbb{R}_{+}^{q} \rightarrow$ $\mathbb{R}: \eta \mapsto\|\eta\|_{1}^{2} / q\|\eta\|^{2}$. It is guaranteed by (7) that the best fairness among UEs is achieved.

$$
J(\mathfrak{\eta})=1
$$

\section{E. Problem Formulation}

The investigated problem is to maximize the QoS by CoMPcell selection and sufficient RU allocation ensured by (5), subject to the capacity/resource limits in (6), and the fairness constraint in (7). The problem is formulated in (8).

$$
\max _{\kappa, \boldsymbol{\alpha}, \boldsymbol{\eta}}\|\eta\|_{1} \quad \text { subject to (3) - (7) }
$$

\section{Computational CompleXity}

Theorem 1. The problem in (8) is $\mathcal{N} \mathcal{P}$-hard.

Proof. We prove the theorem by a polynomial-time reduction from the 3-satisfiability (3-SAT) problem that is $\mathcal{N} \mathcal{P}$-complete. Consider a 3-SAT problem with $\mathrm{N}_{1}$ Boolean variables $\mathrm{b}_{1}, \mathrm{~b}_{2}$, $\ldots, b_{N_{1}}$, and $\mathrm{N}_{2}$ clauses. A Boolean variable or its negation is referred to as a literal, e.g. $\hat{b}_{i}$ is the negation of $b_{i}$. A clause is composed by a disjunction of exactly three distinct literals, e.g. $\left(b_{1} \vee b_{2} \vee \hat{b}_{3}\right)$ is an example of clause. The 3SAT problem amounts to determining whether or not there exists an assignment of true/false to the variables, such that all clauses would be satisfied. To make the reduction from 3-SAT to our problem in (8), we construct a specific network scenario as follows. Suppose we have $\mathrm{N}_{1}+\mathrm{N}_{2}+1$ UEs in total, denoted by $u_{0}, u_{1}, u_{2}, \ldots, u_{N_{1}+N_{2}}$, respectively. Also, we have in total $2 \mathrm{~N}_{1}+\mathrm{N}_{2}$ BSs, denoted by $\mathrm{a}_{1}, \mathrm{a}_{1}^{\prime}, \mathrm{a}_{2}, \mathrm{a}_{2}^{\prime}, \ldots \mathrm{a}_{\mathrm{N}_{1}}, \mathrm{a}_{\mathrm{N}_{1}}^{\prime}$, and $A_{0}, A_{N_{1}+1}, A_{N_{1}+2}, \ldots, A_{N_{1}+N_{2}}$, respectively. For each $u_{i}$ $\left(1 \leqslant i \leqslant N_{1}\right)$, we set $\mathcal{R}_{\ell\left(u_{i}\right)}=\left\{a_{i}, a_{i}^{\prime}\right\}$. For each $u_{j}\left(N_{1}<\right.$ $\left.j \leqslant N_{1}+N_{2}\right)$, we set $\mathcal{R}_{\ell\left(u_{j}\right)}=\left\{A_{j}\right\}$. Besides, $\mathcal{R}_{\ell\left(u_{0}\right)}$ is set to $\left\{A_{0}\right\}$. Let $p_{A_{0}}=N_{1}+1$. For $1 \leqslant i \leqslant N_{1}$ and $N_{1}<j \leqslant$
$\mathrm{N}_{1}+\mathrm{N}_{2}$, let $\mathrm{p}_{\mathrm{a}_{i}}=\mathrm{p}_{\mathrm{A}_{j}}=1$. For simplicity, we use the term "gain value" to refer to $\left|\mathbf{h}_{i j}^{\mathrm{H}} \boldsymbol{w}_{i}\right|$ shown in (2). For any UE $u_{i}$ $\left(1 \leqslant i \leqslant N_{1}+N_{2}\right)$, the gain values of $a_{i}$ and $a_{i}^{\prime}$ equal to 1.0. For UE $u_{0}$, the gain values of all $a_{i}$ and $a_{i}^{\prime}\left(1 \leqslant i \leqslant N_{1}\right)$ equal to 1.0. Besides, the vector $\mathbf{h}_{\mathrm{a}_{i}, \mathfrak{u}_{i}}^{\mathrm{H}} \boldsymbol{w}_{\mathrm{a}_{i}}$ is orthogonal to $\mathbf{h}_{\hat{a}_{i}, u_{i}}^{\mathrm{H}} \boldsymbol{w}_{\hat{a}_{i}}$ for $1 \leqslant i \leqslant N_{1}$. For any $j\left(1 \leqslant j \leqslant N_{1}\right), u_{N_{1}+j}$ has the gain value 1.0 from the BSs that represent the literals in clause $j$. The gain value from $A_{0}$ to $u_{0}$ is 1.0. In addition, from $A_{i}$ to $u_{i}\left(N_{1}<i \leqslant N_{1}+N_{2}\right)$ ), the gain value is 3.0. Gain values between any other BS-UE pair are negligible. The noise power $\sigma^{2}$ is 1.0. In addition, the total traffic demand within the time period $\mathrm{T}$ for each UE is 1.0. We normalize the data traffic of UEs within the time period $T$ by $B \times M$, and the normalized demands are uniformly set to 1.0. The fronthaul capacity is set to be sufficient with respect to this user demand.

First, we note that each UE $j\left(0 \leqslant j \leqslant N_{1}+N_{2}+1\right)$ should be served by at least one $\mathrm{BS}$, otherwise $C_{j}$ equals to 0 and the constraint (5) would be violated. Thus, $A_{0}$ is serving $u_{0}$ and $A_{N_{1}+1}, A_{N_{1}+2}, \ldots, A_{N_{1}+N_{2}}$ are serving $u_{N_{1}+1}, u_{N_{1}+2}, \ldots, u_{N_{1}+N_{2}}$, respectively. Second, it can be verified that $u_{i}\left(1 \leqslant i \leqslant N_{1}\right)$ can only be served by exactly one BS in $\mathcal{R}_{\ell\left(u_{i}\right)}=\left\{a_{i}, \hat{a}_{i}\right\}$. This is because, if $u_{i}$ is served by both $a_{i}$ and $\hat{a}_{i}$, then the BS $A_{0}$ would be overloaded $\left(\rho_{A_{0}}>1\right)$ due to the interference received from all other BSs, and thus the maximum resource limit constraint in (6) would be violated. Besides, for each clause, the three corresponding cells (e.g. for a clause $\left(b_{1} \vee b_{2} \vee \hat{b}_{3}\right)$ the corresponding cells are $a_{1}, a_{2}$ and $\hat{a}_{3}$ ) cannot be all active in serving UEs. Otherwise, the cell that is serving the UE corresponding to this clause would be overloaded, thus violating the maximum resource limit constraint in (6).

Now suppose there is an association that is feasible. For each Boolean variable $b_{i}$, we set $b_{i}=$ true if $a_{i}^{\prime}$ is serving UE $u_{i}$. Otherwise, $u_{i}$ must be served by $a_{i}$ and we set $b_{i}=$ false. For each clause, it is satisfied only if at least one of its literals is with the value true. As discussed above, a feasible solution of the constructed problem cannot have all the corresponding three BSs been in the status of serving UEs, which means that we have at least one of the three BSs been in the idle status. Therefore, a feasible solution of the constructed problem is corresponding to the 3 -SAT problem instance. Hence the conclusion.

\section{SOlution ApproACH}

We show theoretical insights of the joint optimization problem on cell selection and resource allocation in this section. We provide a scheme to achieve global optimal BS resource allocation, with any fixed BS-UE association pattern. Furthermore, we derive partial optimality condition for CoMP-cell selection. An algorithm for solving the problem in (8) is then proposed, based on the theoretical foundations mentioned above.

\section{A. Optimal Resource Allocation}

For clarity, we define the following notations, which are used throughout the remaining of this paper.

Notation 2. For any mapping $\mathcal{M}: \mathbb{R}_{+}^{q} \rightarrow \mathbb{R}_{+}^{q}: \alpha_{\geqslant 0} \mapsto$ $\mathcal{M}\left(\alpha_{\geqslant 0}\right)$, denote $\mathcal{M}^{(k+1)}=\mathcal{M}^{(k)} \circ \mathcal{M}$ for any $k \geqslant 1$. 
Notation 3. Denote the mapping $\mathcal{T}_{\kappa, j}: \mathbb{R}_{+}^{q+1} \rightarrow \mathbb{R}_{+}:\left[\alpha, \eta_{j}\right] \mapsto$ $\int_{T} \eta_{j} t_{j}(\tau) / C_{j}(\alpha, \kappa) d \tau$.

Notation 4. Denote the mapping $\mathcal{H}_{\mathrm{K}}: \mathbb{R}_{+}^{\mathrm{q}+1}: \rightarrow \mathbb{R}_{+}^{\mathrm{q}}:\left[\boldsymbol{\alpha}, \eta_{\mathrm{j}}\right] \rightarrow$ $\left[\mathcal{T}_{\kappa, 1}\left(\alpha, \eta_{j}\right), \mathcal{T}_{\kappa, 2}\left(\alpha, \eta_{j}\right), \ldots, \mathcal{T}_{\kappa, q}\left(\alpha, \eta_{j}\right)\right]^{\top}$.

Notation 5. Denote by $\left\|\alpha_{\geqslant 0}\right\|_{\kappa}$ a norm of $\kappa$ with respect to $\kappa$, such that $\left\|\alpha_{\geqslant 0}\right\|_{\kappa}=\left\|\kappa \alpha_{\geqslant 0}\right\|_{\infty}, \alpha_{\geqslant 0} \in \mathbb{R}_{+}^{q}$.

Theorem 6 provides solution for achieving the optimal resource allocation under any BS-UE association $\kappa^{\prime}$. Before giving the proof, we derive several lemmas, providing theoretical insights of (9), (10), and (11). Theorem 6 is then proved based on these lemmas.

Theorem 6 (Optimal Resource Allocation). For any $\boldsymbol{\kappa}^{\prime} \in$ $\{0,1\}^{\mathrm{m} \times \mathrm{q}}$, consider the mapping $\alpha^{*}:\{0,1\}^{\mathrm{m} \times \mathrm{q}} \rightarrow \mathbb{R}_{++}^{\mathrm{q}}:$ $\kappa^{\prime} \mapsto \arg \max _{\alpha}\left\{\|\eta\|_{1}:(5)-(7), \kappa=\kappa^{\prime}\right\}$. Then

$$
\alpha_{j}^{*}\left(\boldsymbol{\kappa}^{\prime}\right)=\min \left\{\alpha_{j}\left(\boldsymbol{\kappa}^{\prime}\right), \hat{\alpha}_{\mathbf{j}}\left(\boldsymbol{\kappa}^{\prime}\right)\right\} \quad j \in \mathcal{j}
$$

where

$$
\alpha_{\mathbf{j}}\left(\boldsymbol{\kappa}^{\prime}\right)=\lim _{k \rightarrow \infty} \bar{\rho} \mathcal{T}_{\boldsymbol{\kappa}^{\prime}, \mathbf{j}}^{(\mathrm{k})}\left(\boldsymbol{\alpha}_{\geqslant 0}, 1\right) /\left\|\mathcal{H}_{\boldsymbol{\kappa}^{\prime}}^{(\mathrm{k})}\left(\boldsymbol{\alpha}_{\geqslant 0}, 1\right)\right\|_{\boldsymbol{\kappa}^{\prime}}
$$

and

$$
\hat{\alpha}_{j}\left(\kappa^{\prime}\right)=\lim _{k \rightarrow \infty} \mathcal{T}_{\kappa^{\prime}, j}^{(k)}\left(\alpha_{\geqslant 0}, v\right)
$$

with $v=\min _{i \in \mathcal{R}}\left\{c_{i} / \int_{T} \sum_{j \in \mathcal{J}_{i}} t_{j}(\tau) d \tau\right\}$ and $\alpha_{\geqslant 0} \in \mathbb{R}_{+}^{q}$.

Lemma 7. The limits in (10) and (11) exist.

Proof. The proof for (11) is based on the conclusions that 11 falls into the category of standard interference function (SIF) [14], with respect to the variable $\alpha_{\geqslant 0}$, as proved in [15]. An SIF converges to a fixed point that is unique. The proof for (10) is based on Theorem 1 in [16], that the normalized mapping $\mathcal{H}_{\boldsymbol{K}^{\prime}}\left(\boldsymbol{\alpha}_{\geqslant 0}, 1\right) /\left\|\mathcal{H}_{\boldsymbol{K}^{\prime}}\left(\boldsymbol{\alpha}_{\geqslant 0}, 1\right)\right\|_{\boldsymbol{K}^{\prime}}$ converges to a unique fixed point (and thus $\mathcal{T}_{\boldsymbol{K}^{\prime}, j}\left(\boldsymbol{\alpha}_{\geqslant 0}, 1\right) /\left\|\mathcal{H}_{\boldsymbol{K}^{\prime}}\left(\boldsymbol{\alpha}_{\geqslant 0}, 1\right)\right\|_{\boldsymbol{K}^{\prime}}$ for $j \in$ J). Following the conclusion above, the fixed points of $\alpha_{\mathfrak{j}}\left(\boldsymbol{\kappa}^{\prime}\right)$ and $\hat{\alpha}_{\mathfrak{j}}\left(\kappa^{\prime}\right)$ are computed by (10) and (11), respectively.

Lemma 8. $\left\|\boldsymbol{\rho}\left(\boldsymbol{\alpha}\left(\boldsymbol{\kappa}^{\prime}\right), \boldsymbol{\kappa}^{\prime}\right)\right\|_{\infty}=\bar{\rho}$.

Proof. Suppose fixp $\in \mathbb{R}_{+}^{q}$ is the fixed point of $\mathcal{T}_{\kappa^{\prime}, \mathrm{j}}(\operatorname{var}, 1) /\left\|\mathcal{H}_{\boldsymbol{K}^{\prime}}(\operatorname{var}, 1)\right\|_{\kappa^{\prime}}$, with respect to var. By Theorem 1 in [16], there exists $\lambda>0$, such that $\mathcal{T}_{\kappa^{\prime}, j}($ fixp, 1$)=$ $\lambda$ fixp, with $\|$ fixp $\|_{K^{\prime}}=1$. Thus, for the function $\mathcal{T}_{\boldsymbol{K}^{\prime}, \mathrm{j}}(\operatorname{var}, 1) /\left\|\mathcal{H}_{\boldsymbol{\kappa}^{\prime}}(\operatorname{var}, 1) \frac{1}{\bar{\rho}}\right\|_{\boldsymbol{\kappa}^{\prime}}$, we have $\left\|\frac{1}{\bar{\rho}} \boldsymbol{\alpha}\left(\boldsymbol{\kappa}^{\prime}\right)\right\|_{\boldsymbol{\kappa}^{\prime}}=1$ at its fixed point $\alpha\left(\boldsymbol{\kappa}^{\prime}\right)$, which leads to $\left\|\boldsymbol{\kappa}^{\prime} \boldsymbol{\alpha}\left(\boldsymbol{\kappa}^{\prime}\right)\right\|=\bar{\rho}$. By the definition of the mapping $\bar{\rho}$ in Section III we have $\left\|\boldsymbol{\rho}\left(\boldsymbol{\alpha}\left(\boldsymbol{\kappa}^{\prime}\right), \boldsymbol{\kappa}^{\prime}\right)\right\|_{\infty}=\bar{\rho}$. Hence the conclusion.

Lemma 9. Either $\boldsymbol{\alpha}^{*}\left(\boldsymbol{\kappa}^{\prime}\right)=\boldsymbol{\alpha}\left(\boldsymbol{\kappa}^{\prime}\right)$ or $\boldsymbol{\alpha}^{*}\left(\boldsymbol{\kappa}^{\prime}\right)=\hat{\alpha}\left(\boldsymbol{\kappa}^{\prime}\right)$.

Proof. Suppose fixp $\in \mathbb{R}_{+}^{q}$ is the fixed point of $\mathcal{T}_{\kappa^{\prime}, j}(\operatorname{var}, 1) /\left\|\mathcal{H}_{\kappa^{\prime}}(\operatorname{var}, 1)\right\|_{\kappa^{\prime}}$, with respect to var. By Theorem 1 in [16]), there exists $\lambda>0$, such that $\mathcal{T}_{\kappa^{\prime}, j}($ fixp, 1$)=$ $\lambda$ fixp, with $\| \frac{1}{\bar{\rho}}$ fixp $\|_{\kappa^{\prime}}=1$. We then conclude that, if in the limit in (11) we have exactly $v=\lambda$, then $\hat{\alpha}\left(\kappa^{\prime}\right)=\alpha\left(\kappa^{\prime}\right)$. For clarity in the following proof, we denote this specific $\hat{\alpha}\left(\kappa^{\prime}\right)$ by $\hat{\alpha}\left(\kappa^{\prime}, \lambda\right)$. Now consider any $v$ with $v>\lambda$. We look into the corresponding sequence of the limit in (11).
For any $k \geqslant 1$ and $j \in \mathcal{J}$, denote $\alpha_{j}^{(k)}=\mathcal{T}_{\boldsymbol{k}^{\prime}, j}\left(\boldsymbol{\alpha}^{(\mathrm{k}-1)}, \boldsymbol{v}\right)$, with $\alpha^{(0)}=\alpha\left(\kappa^{\prime}, \lambda\right)$. Note that $\alpha_{j}^{(0)}=\mathcal{T}_{\kappa^{\prime}, j}\left(\alpha^{(0)}, \lambda\right)$ for any $j \in \mathcal{J}$. By our construction, the sequence $\alpha^{(0)}, \alpha^{(1)}, \ldots, \alpha^{(\infty)}$ converges to $\alpha\left(\kappa^{\prime}\right)$, i.e. $\alpha^{(\infty)}=\alpha\left(\kappa^{\prime}\right)$. By our definition of the sequence above, $\alpha_{j}^{(1)}=\mathcal{T}_{\boldsymbol{K}^{\prime}, \mathrm{j}}\left(\boldsymbol{\alpha}^{(0)}, v\right)$. Meanwhile, note that for any $j \in \mathcal{J}, \mathcal{T}_{\kappa^{\prime}, j}\left(\alpha^{(0)}, v\right) \geqslant \mathcal{T}_{\kappa^{\prime}, j}\left(\alpha^{(0)}, \lambda\right)$ holds, due to that $\mathcal{T}_{\boldsymbol{K}^{\prime}, j}\left(\boldsymbol{\alpha}^{(0)}, \boldsymbol{v}\right)$ is monotonic in $v($ or $\lambda)$. Therefore, $\boldsymbol{\alpha}^{(1)} \geqslant \boldsymbol{\alpha}^{(0)}$. By using the same way, one can prove that $\alpha^{(\infty)} \geqslant \alpha^{(0)}$, and hence $\hat{\alpha}\left(\boldsymbol{\kappa}^{\prime}\right) \geqslant \boldsymbol{\alpha}\left(\boldsymbol{\kappa}^{\prime}\right)$. Similarly, for any $\boldsymbol{v}<\lambda$, we can prove that $\hat{\alpha}\left(\kappa^{\prime}\right) \leqslant \boldsymbol{\alpha}\left(\kappa^{\prime}\right)$ holds. Hence the conclusion.

Lemma 10. Denote $\eta^{*}\left(\boldsymbol{\kappa}^{\prime}\right)=\arg \max _{\boldsymbol{\eta}}\left\{\|\boldsymbol{\eta}\|_{1}:\right.$ (5), (6), $\boldsymbol{\alpha}=$ $\left.\alpha^{*}\left(\boldsymbol{\kappa}^{\prime}\right), \boldsymbol{\kappa}=\boldsymbol{\kappa}^{\prime}\right\}$. Then $\eta_{1}^{*}\left(\boldsymbol{\kappa}^{\prime}\right)=\eta_{2}^{*}\left(\boldsymbol{\kappa}^{\prime}\right)=\cdots=\eta_{\mathrm{q}}^{*}\left(\boldsymbol{\kappa}^{\prime}\right)$.

Proof. We first note that the feasibility holds regarding the constraints in the optimization problem stated in this lemma, by setting $\eta_{1}=\eta_{2}=\cdots=\eta_{q}=0$. The we prove that at its optimum we have $\eta_{1}=\eta_{2}=\cdots=\eta_{\mathrm{q}}$. By Lemma 9 , we either have $\boldsymbol{\alpha}^{*}\left(\boldsymbol{\kappa}^{\prime}\right)=\boldsymbol{\alpha}\left(\boldsymbol{\kappa}^{\prime}\right)$ or $\boldsymbol{\alpha}^{*}\left(\boldsymbol{\kappa}^{\prime}\right)=\hat{\boldsymbol{\alpha}}\left(\boldsymbol{\kappa}^{\prime}\right)$. We first consider the case of $\boldsymbol{\alpha}^{*}\left(\boldsymbol{\kappa}^{\prime}\right)=\hat{\alpha}\left(\boldsymbol{\kappa}^{\prime}\right)$. In this case, we have for any $j \in \mathcal{J}$ that $\eta_{j} \leqslant \hat{\alpha}_{j}\left(\kappa^{\prime}\right) C_{j}\left(\hat{\alpha}\left(\kappa^{\prime}\right), \kappa^{\prime}\right) / \int_{T} t_{j}(\tau) d \tau$, by the constraint (5). Combined with (11) that $v=$ $\hat{\alpha}_{j}\left(\kappa^{\prime}\right) C_{j}\left(\hat{\alpha}\left(\kappa^{\prime}\right), \kappa^{\prime}\right) / \int_{T} t_{j}(\tau) d \tau(j \in \mathcal{J})$, we then have $\eta_{j} \leqslant$ $v=\min _{i \in \mathcal{R}}\left\{c_{i} / \int_{T} \sum_{j \in \mathcal{J}_{i}} t_{j}(\tau) d \tau\right\}$ for any $j \in \mathcal{J}$. Also, note that $\eta_{j} \leqslant v$ with all $j \in \mathcal{J}$ indicates that the fronthaul capacity constraint in (6) is satisfied. In addition, by the definition of $\boldsymbol{\alpha}^{*}\left(\boldsymbol{\kappa}^{\prime}\right)$ in 9 , we have $\boldsymbol{\alpha}^{*}\left(\boldsymbol{\kappa}^{\prime}\right) \leqslant \boldsymbol{\alpha}\left(\boldsymbol{\kappa}^{\prime}\right)$, which leads to $\rho\left(\boldsymbol{\alpha}^{*}\left(\boldsymbol{\kappa}^{\prime}\right), \boldsymbol{\kappa}^{\prime}\right) \leqslant \boldsymbol{\rho}\left(\boldsymbol{\alpha}\left(\boldsymbol{\kappa}^{\prime}\right), \boldsymbol{\kappa}^{\prime}\right)$. Along with Lemma 8, we conclude that $\rho_{i}\left(\alpha^{*}\left(\kappa^{\prime}\right), \kappa^{\prime}\right) \leqslant \bar{\rho}$ holds for any $i \in \mathcal{R}$, and hence the maximum resource limit constraint in (6) is satisfied. In this case, we should have $\eta_{1}=\eta_{2}=\cdots=\eta_{\mathrm{q}}=v$ so as to reach the maximum of $\|\eta\|_{1}$.

For the other case that $\alpha^{*}\left(\boldsymbol{\kappa}^{\prime}\right)=\alpha\left(\boldsymbol{\kappa}^{\prime}\right)$, we can verify that the maximum resource limit constraint in (6) is satisfied, by applying Lemma 8 directly. Also, from the proof of Lemma 10 , we have some $\lambda$ such that $\lambda=\alpha_{1}\left(\kappa^{\prime}\right) / \mathcal{T}_{\kappa^{\prime}, 1}\left(\alpha\left(\kappa^{\prime}\right), 1\right)=$ $\alpha_{2}\left(\boldsymbol{\kappa}^{\prime}\right) / \mathcal{T}_{\boldsymbol{\kappa}^{\prime}, 2}\left(\boldsymbol{\alpha}\left(\boldsymbol{\kappa}^{\prime}\right), 1\right)=\cdots=\alpha_{\mathrm{q}}\left(\boldsymbol{\kappa}^{\prime}\right) / \mathcal{T}_{\boldsymbol{\kappa}^{\prime}, \mathrm{q}}\left(\boldsymbol{\alpha}\left(\boldsymbol{\kappa}^{\prime}\right), 1\right)$. Вy the proof in Lemma 9, we know that in this case we have $\lambda<v$ holds, and thus the fronthaul capacity constraint in (6) is satisfied. By the constraint in (5), we have $\eta_{j} \leqslant$ $\alpha_{j}\left(\boldsymbol{\kappa}^{\prime}\right) C_{j}\left(\boldsymbol{\alpha}\left(\boldsymbol{\kappa}^{\prime}\right), \boldsymbol{\kappa}^{\prime}\right) / \int_{\mathrm{T}} t_{j}(\tau) \mathrm{d} \tau=\lambda$. We therefore have $\eta_{1}=\eta_{2}=\cdots=\eta_{\mathrm{q}}=\lambda$ so as to reach the maximum of $\|\eta\|_{1}$.

Lemma 11. For any $j \in \mathcal{J}$ in Lemma 10, 12 holds.

$$
\eta_{\mathfrak{j}}^{*}\left(\boldsymbol{\kappa}^{\prime}\right)=\min \left\{\alpha_{\mathfrak{j}}^{*}\left(\boldsymbol{\kappa}^{\prime}\right) / \mathcal{T}_{\boldsymbol{\kappa}^{\prime}, \mathbf{j}}\left(\boldsymbol{\alpha}^{*}\left(\boldsymbol{\kappa}^{\prime}\right), 1\right), v\right\}
$$

Proof. The proof is directly based on Lemma 9 and 10 and their corresponding proofs. For the two cases $\boldsymbol{\alpha}^{*}\left(\boldsymbol{\kappa}^{\prime}\right)=\boldsymbol{\alpha}\left(\boldsymbol{\kappa}^{\prime}\right)$ and $\boldsymbol{\alpha}^{*}\left(\boldsymbol{\kappa}^{\prime}\right)=\hat{\alpha}\left(\boldsymbol{\kappa}^{\prime}\right)$, note that we have $\eta_{j}=\min \{\lambda, \boldsymbol{v}\}$ respectively, for all $j \in \mathcal{J}$. Since $\lambda=\alpha_{1}\left(\boldsymbol{\kappa}^{\prime}\right) / \mathcal{T}_{\boldsymbol{K}^{\prime}, 1}\left(\boldsymbol{\alpha}\left(\boldsymbol{\kappa}^{\prime}\right), 1\right)=$ $\alpha_{2}\left(\boldsymbol{\kappa}^{\prime}\right) / \mathcal{T}_{\boldsymbol{\kappa}^{\prime}, 2}\left(\boldsymbol{\alpha}\left(\boldsymbol{\kappa}^{\prime}\right), 1\right)=\cdots=\alpha_{\mathrm{q}}\left(\boldsymbol{\kappa}^{\prime}\right) / \mathcal{T}_{\boldsymbol{K}^{\prime}, \mathbf{q}}\left(\boldsymbol{\alpha}\left(\boldsymbol{\kappa}^{\prime}\right), 1\right)$, we reach the conclusion.

\section{The proof of Theorem 6 is as follows:}

Proof. For the problem P1 : $\arg \max _{\alpha}\left\{\|\boldsymbol{\eta}\|_{1}:(5)-(7), \kappa=\kappa^{\prime}\right\}$, we can prove by Lemma 10 that $\alpha=\alpha^{*}\left(\kappa^{\prime}\right)$ satisfies the 
constraint (7), due to that $\eta_{1}^{*}\left(\kappa^{\prime}\right)=\eta_{2}^{*}\left(\kappa^{\prime}\right)=\cdots=\eta_{\mathrm{q}}^{*}\left(\boldsymbol{\kappa}^{\prime}\right)$. Also, $\boldsymbol{\alpha}^{*}\left(\boldsymbol{\kappa}^{\prime}\right)$ is the optimal solution for the problem P2 : $\arg \max _{\boldsymbol{\eta}}\left\{\|\boldsymbol{\eta}\|_{1}:\right.$ (5), (6), $\left.\boldsymbol{\alpha}=\boldsymbol{\alpha}^{*}\left(\boldsymbol{\kappa}^{\prime}\right), \boldsymbol{\kappa}=\boldsymbol{\kappa}^{\prime}\right\}$, according to Lemma 10, which means that $\boldsymbol{\alpha}\left(\boldsymbol{\kappa}^{\prime}\right)$ satisfies the constraints (5) and (6). Therefore, $\alpha\left(\kappa^{\prime}\right)$ is a feasible solution of $\mathrm{P} 1$. Consider there are another feasible solution of $\mathrm{P} 1$, $\left\langle\boldsymbol{\eta}^{\prime}, \boldsymbol{\alpha}^{\prime}\right\rangle$ that leads to an objective value $\left\|\boldsymbol{\eta}^{\prime}\right\|>\left\|\boldsymbol{\eta}^{*}\right\|$. To meet the constraint in (7), we should have $\eta_{1}^{\prime}=\eta_{2}^{\prime}=\cdots \eta_{q}^{\prime}$. Therefore, we have either $\eta_{j}^{\prime}=\beta \lambda$ or $\eta_{j}^{\prime}=\beta \nu(\beta>1$, $j \in \mathcal{J})$. In addition, under the constraints (5) and (6), we should respectively have $\eta_{j}^{\prime} \leqslant \alpha_{j}^{\prime} C_{j}\left(\alpha^{\prime}, \kappa^{\prime}\right) / \int_{T} t_{j}(\tau) d \tau$ and $\eta_{j} \leqslant \min _{i \in \mathcal{R}}\left\{c_{i} / \int_{T} \sum_{j \in \mathcal{J}_{i}} t_{j}(\tau) d \tau\right\}$. It can then be verified that the two constraints cannot be satisfied together by $\eta^{\prime}$, which conflicts our assumption that $\left\langle\boldsymbol{\eta}^{\prime}, \boldsymbol{\alpha}^{\prime}\right\rangle$ is feasible. Hence the conclusion.

\section{B. CoMP-cell Selection}

The partial optimality condition of CoMP-cell selection is given by Theorem [16, which is proved based on Lemma 14 and Lemma 15

Definition 12. For any $\mathfrak{j} \in \mathcal{J}$ and $r \subseteq \mathcal{R}$, define the mapping $\mathcal{E}_{j, g}:\{0,1\}^{\mathrm{m} \times \mathrm{q}} \rightarrow\{0,1\}^{\mathrm{m} \times \mathrm{q}}: \mathbf{\kappa} \rightarrow \hat{\mathbf{k}} \mid \mathcal{J}_{\mathfrak{j}}(\hat{\mathbf{k}})=\mathcal{J}_{\mathfrak{j}}(\mathbf{k}) \cup r$.

Definition 13 (CoMP-cell filter). Denote $\boldsymbol{\kappa}^{\prime \prime}=\mathcal{E}_{\mathfrak{j},\{i\}}\left(\boldsymbol{\kappa}^{\prime}\right)$. For any UE $j$ and any target set $r \subseteq \mathcal{R}$, define the mapping $\{0,1\}^{\mathrm{m} \times \mathrm{q}} \rightarrow\{0,1\}^{\mathrm{m} \times \mathrm{q}}$ as the filter of $r$

$$
\mathcal{F}_{j,\{i\}}: \kappa^{\prime} \mapsto \begin{cases}\kappa^{\prime \prime} & \text { condition satisfied } \\ \kappa^{\prime} & \text { otherwise }\end{cases}
$$

such that the condition is $\rho_{i}\left(\mathcal{H}_{\boldsymbol{\kappa}^{\prime \prime}}\left(\boldsymbol{\alpha}^{*}\left(\boldsymbol{\kappa}^{\prime}\right), \eta_{j}^{*}\right), \boldsymbol{\kappa}^{\prime \prime}\right) \leqslant$ $\rho_{i}\left(\boldsymbol{\alpha}^{*}\left(\boldsymbol{\kappa}^{\prime}\right), \kappa^{\prime \prime}\right)$, where the parameter $\mu_{i}$ is defined as $\mu=$ $\mathcal{T}_{\boldsymbol{\kappa}^{\prime \prime}, \mathrm{j}}\left(\boldsymbol{\alpha}^{*}\left(\boldsymbol{\kappa}^{\prime}\right), \eta_{\mathrm{j}}^{*}\right) / \boldsymbol{\alpha}_{\mathbf{j}}^{*}\left(\boldsymbol{\kappa}^{\prime}\right)$ and the resource allocation $\boldsymbol{\alpha}\left(\boldsymbol{\kappa}^{\prime}\right)$ follows the definition in (9).

Lemma 14. $\mathcal{H}_{\boldsymbol{\kappa}^{\prime \prime}}\left(\boldsymbol{\alpha}^{*}\left(\boldsymbol{\kappa}^{\prime}\right), \eta_{\mathbf{j}}^{*}\left(\boldsymbol{\kappa}^{\prime}\right)\right) \leqslant \boldsymbol{\alpha}^{*}\left(\boldsymbol{\kappa}^{\prime}\right)$.

Proof. Let $\boldsymbol{\kappa}^{\prime \prime}=\mathcal{E}_{\mathfrak{j},\{\mathfrak{i}\}}\left(\boldsymbol{\kappa}^{\prime}\right)$. Then we have $\mathcal{J}_{\mathfrak{j}}\left(\boldsymbol{\kappa}^{\prime}\right) \subset \mathcal{J}_{\mathfrak{j}}\left(\boldsymbol{\kappa}^{\prime \prime}\right)$, which leads to that $\gamma_{j}\left(\kappa^{\prime \prime}\right)>\gamma_{j}\left(\kappa^{\prime}\right)$ according to (2). Therefore, we have $\mathcal{T}_{\boldsymbol{K}^{\prime \prime}, j}\left(\boldsymbol{\alpha}^{*}\left(\boldsymbol{\kappa}^{\prime}\right), \eta_{j}^{*}\right)<\alpha_{j}^{*}\left(\boldsymbol{\kappa}^{\prime}\right)$. Hence the conclusion. Since $J_{k \neq j}\left(\kappa^{\prime \prime}\right)=J_{k \neq j}\left(\kappa^{\prime}\right)$, we conclude that $\mathcal{T}_{\boldsymbol{\kappa}^{\prime \prime}, k \neq j}\left(\boldsymbol{\alpha}^{*}\left(\boldsymbol{\kappa}^{\prime}\right), \eta_{\mathrm{k} \neq \mathrm{j}}^{*}\right)=\alpha_{\mathrm{k} \neq \mathrm{j}}^{*}\left(\boldsymbol{\kappa}^{\prime}\right)$. Hence the conclusion.

Lemma 15. $\lim _{k \rightarrow \infty} \mathcal{H}_{\boldsymbol{\kappa}^{\prime \prime}}^{(\mathrm{k})}\left(\boldsymbol{\alpha}_{\geqslant 0}, \eta_{j}^{*}\left(\boldsymbol{\kappa}^{\prime}\right)\right) \leqslant \boldsymbol{\alpha}^{*}\left(\boldsymbol{\kappa}^{\prime}\right)$ with $\boldsymbol{\alpha}_{\geqslant 0} \in$ $\mathbb{R}_{+}^{q}$.

Proof. Since $\mathcal{H}_{\mathrm{k}^{\prime \prime}}^{(\mathrm{k})}\left(\mathrm{var}, \eta_{j}^{*}\right)$ is monotonic in var, we reach the conclusion by Lemma 14

Theorem 16 (Partial Optimality of $\mathcal{F}_{\mathfrak{j},\{i\}}$ ). For any $\boldsymbol{\kappa}^{\prime}, j \in \mathcal{J}$, and $r \subseteq \mathcal{R}, \max _{\boldsymbol{\alpha}}\left\{\|\boldsymbol{\eta}\|_{1}:(5 \mathbf{5})-(\mathbf{7}), \boldsymbol{\kappa}=\mathcal{F}_{\mathbf{j},\{i\}}\left(\boldsymbol{\kappa}^{\prime}\right)\right\} \geqslant \max _{\boldsymbol{\alpha}}\left\{\|\boldsymbol{\eta}\|_{1}:\right.$ (5) - (7), $\left.\kappa=\kappa^{\prime}\right\}$.

Proof. If $\mathcal{F}_{\mathfrak{j},\{i\}}\left(\boldsymbol{\kappa}^{\prime}\right)=\boldsymbol{\kappa}^{\prime}$, then we have that the equality in the statement holds element-wisely for $\alpha$ in the theorem. For the other case that $\mathcal{F}_{\mathbf{j},\{i\}}\left(\boldsymbol{\kappa}^{\prime}\right)=\boldsymbol{\kappa}^{\prime \prime}$, we have by Lemma 14 that $\eta_{j}^{*}\left(\boldsymbol{\kappa}^{\prime}\right) \leqslant \boldsymbol{\alpha}^{*}\left(\boldsymbol{\kappa}^{\prime}\right) / \mathcal{T}_{\boldsymbol{\kappa}^{\prime \prime}, \mathrm{j}}\left(\boldsymbol{\alpha}^{*}\left(\boldsymbol{\kappa}^{\prime}\right), 1\right)$. Thus all the active constraints in (5) and (6) are relaxed. Combined with Lemma 15. we have $\eta_{j}\left(\boldsymbol{\kappa}^{\prime \prime}\right) \geqslant \eta_{\mathfrak{j}}\left(\boldsymbol{\kappa}^{\prime}\right)$. Hence the conclusion.

\section{Algorithm Design}

The proposed algorithm is shown in Algorithm 1, of which the time-consuming part is on computing the convergence point $\alpha^{*}(\kappa)$ of equation (10) and (11), with respect to the CoMP association pattern $\kappa$. As stated in Line 2, the fixedpoint iterations are done with $\sum_{j=1}^{q}\left|\mathcal{R}_{(\ell(j))}\right|$ rounds. Suppose the time-complexity of the algorithm for computing the fixed point of (10) and (11) is in $\mathrm{O}(\mathrm{K})$. Then the time-complexity of Algorithm 1 is in $\mathrm{O}(\mathrm{qmK})$.

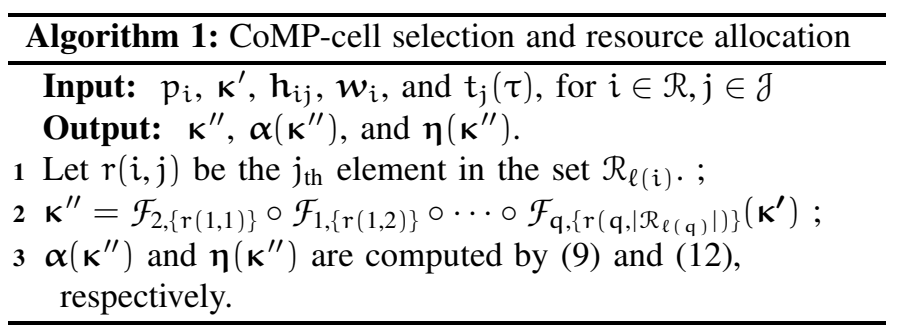

\section{Simulation}

We deploy 3 C-RAN clusters with hexagonal coverage region (500 meters radius). There is an RCC located in the center of each hexagon, along with a BBU pool. In each hexagonal region, several BSs are deployed with an RRH. The RRH is connected with a Common Public Radio Interface (CPRI) based fronthaul of 2.5 Gbps capacity limit to each RRH [17]. Multiple UEs are randomly and uniformly distributed in each hexagonal region. The network operates at $2 \mathrm{GHz}$. Each RU is set to $180 \mathrm{KHz}$ bandwidth and the bandwidth for each cell is $20 \mathrm{MHz}$. We remark that the simulation setting of bandwidth follows the 3GPP standardization document [18]. The noise power spectral density is set to $-174 \mathrm{dBm} / \mathrm{Hz}$. The transmit power of BSs on each RU is $200 \mathrm{~mW}$. The path loss between BS and UE follows the standard 3GPP micro models [19]. The shadowing coefficients are generated by the log-normal distribution with $3 \mathrm{~dB}$ standard deviation [19].

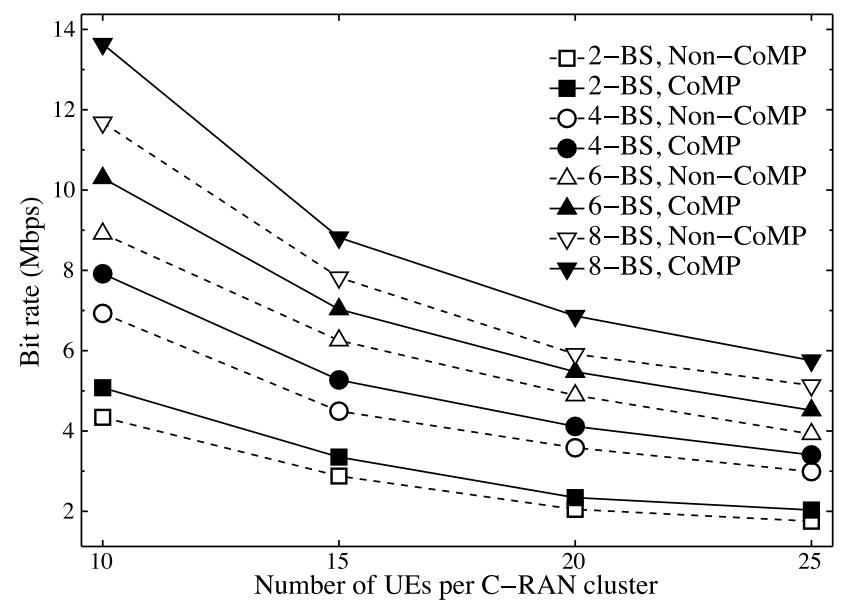

Figure 2. Bit rate vs. number of UEs $(\bar{\rho}=1.0)$.

In the numerical results below, we compare the QoS performance of CoMP with that of non-CoMP case. In both cases, the 
time-frequency resource allocation is optimized by Theorem 6 The evaluation is done under the scenarios with different number of BSs, UEs, and values of the maximum resource limit $\bar{\rho}$. Figure 2 shows the QoS performance with respect to different amount of UEs and BSs in each C-RAN cluster. We deploy respectively $2,4,6,8 \mathrm{BSs}$ (RRHs) that are capable to cooperatively serve UEs via CoMP within each C-RAN cluster. Intuitively, the QoS depends on user density and resource sharing. Numerically, the QoS decreases with the increase of UEs' density. Besides, the QoS can be enhanced by deploying more cooperative RRHs in a C-RAN cluster. Compared to the non-CoMP case, the QoS performance always benefits from optimizing the CoMP-cell selection. When UEs are densely distributed, one can achieve almost the same QoS enhancement by CoMP within C-RAN cluster, as by increasing the BS density. With the increase of BS density, the UEs gain more on QoS improvement. On average, the QoS is improved via CoMP by $11.6 \%$. In Figure 3 , we compare the QoS performance for the cases with different maximum available resource constraint, i.e. $\bar{\rho}$. The network benefits more via CoMP with a larger value of $\bar{\rho}$. That means, the cooperation among BSs via CoMP would be crucial, with sufficient available time-frequency resource in the network. On average, the QoS improvement of CoMP can reach to $11.3 \%$.

In general, the possible improvement on QoS through CoMP and resource allocation is sensitive to the network density (for both BS and UE) as well as the resource limit in each cell. In $5 \mathrm{G}$, the network is likely to be ultra-densely deployed with BSs (e.g. small/femto stations), resulting in that there could be more stations than UEs in a cell coverage of $5 \mathrm{G}$ cellular networks. Further, as the mobile systems of 5G are broadening their spectrum, the available resource would be more sufficient in the next generation networks.

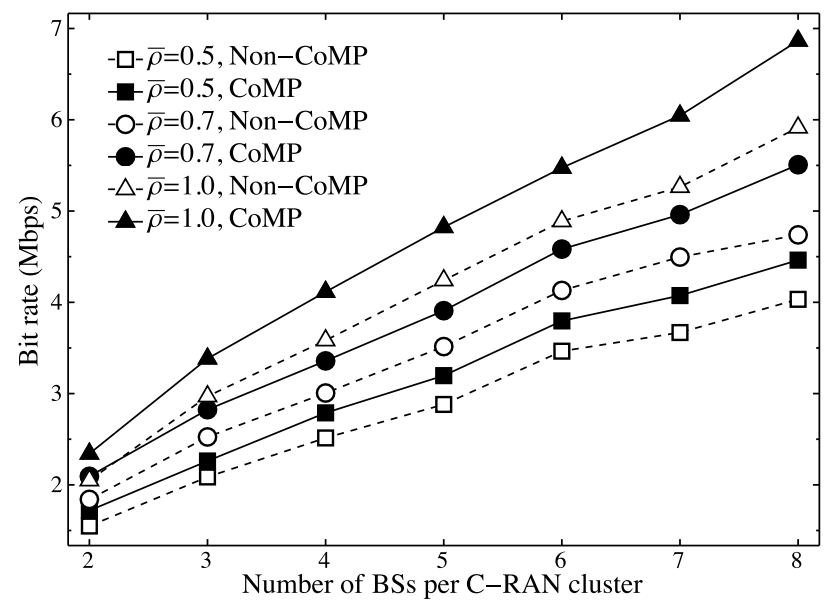

Figure 3. Bit rate vs. number of BSs (20 UEs per cluster).

\section{CONCLUSION}

For the joint CoMP-cell selection and resource allocation problem with fronthaul-constrained C-RAN, theoretical analysis regarding the computational complexity has been given.
A joint optimization problem of cell selection and resource allocation has been proposed.

\section{ACKNOWLEDGEMENT}

This work has been supported by the Swedish Research Council and the Linköping-Lund Excellence Center in Information Technology (ELLIIT), Sweden, and the European Union Marie Curie project MESH-WISE (FP7-PEOPLE2012-IAPP: 324515), DECADE (H2020-MSCA-2014-RISE: 645705), and WINDOW (FP7-MSCA-2012-RISE: 318992). The work of D. Yuan has been carried out within European FP7 Marie Curie IOF project 329313.

\section{REFERENCES}

[1] "Cloud ran - the benefits of virtualization, centralization and coordination,” Ericsson, Tech. Rep., Sep. 2015.

[2] M. Peng, Y. Li, Z. Zhao, and C. Wang, "System architecture and key technologies for $5 \mathrm{~g}$ heterogeneous cloud radio access networks," IEEE network, vol. 29, no. 2, pp. 6-14, 2015.

[3] M. Peng, C. Wang, V. Lau, and H. V. Poor, "Fronthaul-constrained cloud radio access networks: insights and challenges," IEEE Wireless Communications, vol. 22, no. 2, pp. 152-160, 2015.

[4] "White paper of next generation fronthaul interface," China Mobile Research Institute, Tech. Rep. V1.0, Jun. 2015.

[5] "Cloud-ran deploymnet with cpri fronthaul technology," VIAVI, Tech. Rep.

[6] M. Artuso and H. Christiansen, "Fronthaul dimensioning in c-ran with web traffic for coordinated multipoint joint transmission," 2015, pp. 5055.

[7] H. Zhang, C. Jiang, J. Cheng, and V. C. M. Leung, "Cooperative interference mitigation and handover management for heterogeneous cloud small cell networks," IEEE Wireless Communications, vol. 22, no. 3, pp. 92-99, Jun. 2015.

[8] A. Davydov, G. Morozov, I. Bolotin, and A. Papathanassiou, "Evaluation of joint transmission comp in c-ran based lte-a hetnets with large coordination areas," in Proceedings of IEEE Globecom Workshops, 2014, pp. 801-806.

[9] A. Beylerian and T. Ohtsuki, "Multi-point fairness in resource allocation for c-ran downlink comp transmission," EURASIP Journal on Wireless Communications and Networking, vol. 2016, no. 1, pp. 1-10, 2016.

[10] M. Peng, Y. Li, J. Jiang, J. Li, and C. Wang, "Heterogeneous cloud radio access networks: a new perspective for enhancing spectral and energy efficiencies," IEEE Wireless Communications, vol. 21, no. 6, pp. 126135,2014

[11] J. Ortín, J. R. Gállego, and M. Canales, "Joint cell selection and resource allocation games with backhaul constraints," Pervasive and Mobile Computing, 2016.

[12] A. Abdelnasser and E. Hossain, "Resource allocation for an ofdma cloudran of small cells underlaying a macrocell," IEEE Transactions on Mobile Computing, vol. 15, no. 11, pp. 2837-2850, 2016.

[13] G. Nigam, P. Minero, and M. Haenggi, "Coordinated Multipoint Joint Transmission in Heterogeneous Networks," IEEE Transactions on Communications, vol. 62, no. 11, pp. 4134-4146, 2014.

[14] R. D. Yates, "A framework for uplink power control in cellular radio systems," IEEE Journal on Selected Areas in Communications, vol. 13, no. 7, pp. 1341-1347, 1995.

[15] L. You and D. Yuan, "Load optimization with user association in cooperative and load-coupled lte networks," arXiv.org, 2016.

[16] U. Krause, "Concave perron-frobenius theory and applications," Nonlinear Analysis: Theory, Method \& Applications, 2001.

[17] K. Murphy, "Centralized ran and fronthaul," Ericsson, Tech. Rep., May 2015.

[18] 3GPP, Tech. Rep. TR 36.116,V13.0.1, 2016.

[19] "Evolved universal terrestrial radio access (e-utra); further advancements for e-utra physical layer aspects," Tech. Rep. 